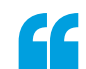

\section{The NMR methods allow electrolyte decomposition and battery self-discharge to be explored in real time}

\title{
Batteries under radical scrutiny
}

Redox flow batteries are typically less hazardous and cheaper than other batteries but their lifetimes - particularly those using organic redox-active electrolytes - are often shorter. Improving the lifetimes and performances of redox flow batteries requires knowledge of the pathways causing decomposition and self-discharge of the cells, as well as identification of the decomposition products.

Now, writing in Nature, Clare Grey and colleagues have devised simple-to-use NMR setups to investigate the electrochemical processes in organic redox flow batteries. These setups - which either monitor the electrolytes flowing out of the cell or probe the electrolytes inside the cell - reveal mechanisms and phenomena responsible for aiding and harming the function of the battery. The batteries probed by the researchers comprise quinonebased electrolytes, for example, 2,6-dihydroxyanthraquinone (DHAQ).

"The NMR methods allow electrolyte decomposition and

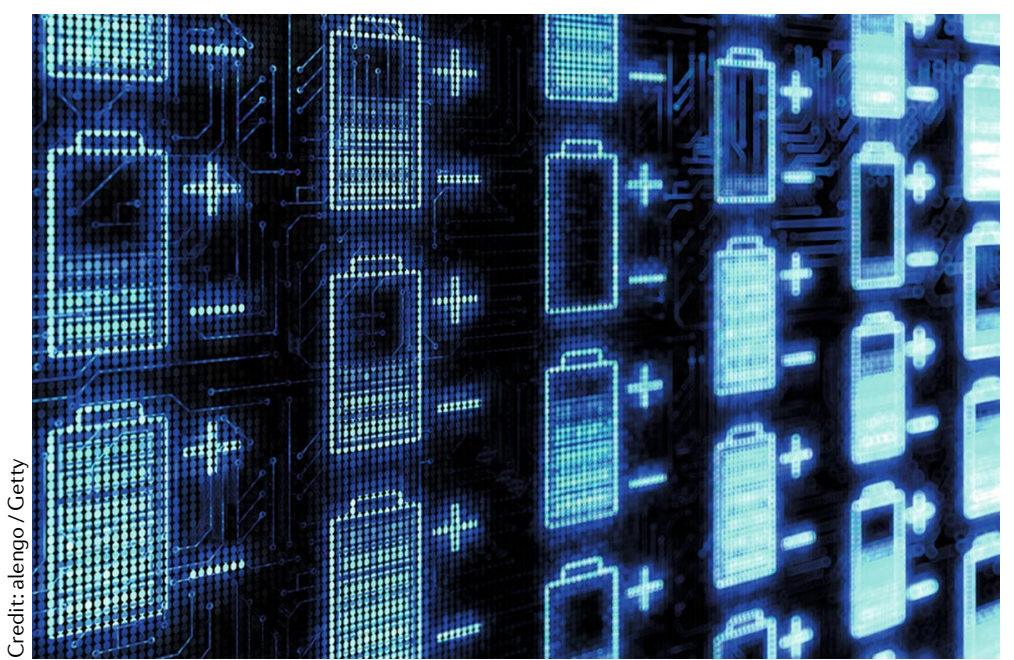

battery self-discharge to be explored in real time," says Grey. "The measurements show that DHAQ is decomposed electrochemically via a reaction which can be minimized by limiting the voltage used on charging."

The NMR method, which probes the electrolyte as it flows out of the battery, reveals useful information about the battery by measuring ${ }^{1} \mathrm{H}$ NMR shifts. By monitoring the change in the shift of the water resonance, the bulk magnetic susceptibility is measured and, as a result, the concentration of radicals in solution can be estimated. "Also, by measuring the line-broadening of the ${ }^{1} \mathrm{H}$ shifts of the quinone resonances as a function of state of charge, we identify and quantify the rate of electron transfer between reduced and oxidised species, and the extent of electron delocalization over the radical anions," says Grey.

A deeper understanding of the self-discharge of the flow battery is gained by combining the NMR method with in situ mass spectrometry. This investigation shows that hydrogen is evolved during the self-discharge process, further indicating the instability of anthraquinone-water mixtures at low potentials. "These findings call for further investigation of anthraquinone degradation processes in the presence of water," says Grey.

The researchers envisage combining in situ NMR techniques with other characterization methods, including electron paramagnetic resonance (EPR) and optical methods. "The goal is to achieve multi-modal in situ characterisation using these highly complementary techniques," says Grey. "For example, NMR studies diamagnetic species while EPR studies the paramagnetic species; and optical methods, such as UV-Vis reveal the electronic structures of molecules." Bringing these techniques together should enable the reactivity and stability of both organic and inorganic redox-active species as well as other non-active components of flow batteries, such as salts and solvent, to be easily assessed.

Although not as easy to practically achieve compared with flow batteries, this online monitoring system may be used to investigate static batteries, such as lithium-air and lithium-ion batteries. "Beyond battery research, these techniques will be applicable to study reaction equilibrium and kinetics in many electrocatalytic systems, for example, electrochemical desalination and the electrochemical reduction and sequestration of $\mathrm{CO}_{2}$, concludes Grey.

Alison Stoddart ORIGINAL ARTICLE Zhao, E. W. et al. In situ NM metrology reveals reaction mechanisms in redox flow batteries. Nature 579, 224-228 (2020) 